\title{
Burn mortality in patients with preexisting cardiovascular disease
}

\author{
Laquanda Knowlin, Trista Reid, Felicia Williams, Bruce Cairns, and Anthony Charles* \\ Department of Surgery, University of North Carolina at Chapel Hill, North Carolina Jaycee Burn \\ Center, United States
}

\begin{abstract}
Introduction-Burn shock, a complex process, which develops following burn leads to severe and unique derangement of cardiovascular function. Patients with preexisting comorbidities such as cardiovascular diseases may be more susceptible. We therefore sought to examine the impact of preexisting cardiovascular disease on burn outcomes.
\end{abstract}

Methods-A retrospective analysis of patients admitted to a regional burn center from 2002 to 2012. Independent variables analyzed included basic demographics, burn mechanism, presence of inhalation injury, TBSA, pre-existing comorbidities, and length of ICU/hospital stay. Bivariate analysis was performed and Poisson regression modeling was utilized to estimate the incidence of being in the ICU and mortality.

Results-There were a total of 5332 adult patients admitted over the study period. $6 \%(\mathrm{n}=428)$ had a preexisting cardiovascular disease. Cardiovascular disease patients had a higher mortality rate $(16 \%)$ compared to those without cardiovascular disease $(3 \%, \mathrm{p}<0.001)$. The adjusted Poisson regression model to estimate incidence risk of being in intensive care unit in patients with cardiovascular disease was 33\% higher compared to those without cardiovascular disease (IRR $=1.33,95 \% \mathrm{CI}=1.22-1.47$ ). The risk for mortality is $42 \%$ higher $(\mathrm{IRR}=1.42,95 \% \mathrm{CI}=1.10$ 1.84) for patients with pre-existing cardiovascular disease compared to those without cardiovascular disease after controlling for other covariates.

Conclusion-Preexisting cardiovascular disease significantly increases the risk of intensive care unit admission and mortality in burn patients. Given the increasing number of Americans with cardiovascular diseases, there will likely be a greater number of individuals at risk for worse outcomes following burn. This knowledge can help with burn prognostication.

\section{Keywords}

Burn; Cardiovascular disease; Comorbidities; Burn mortality; Burn prognostication

\footnotetext{
*Corresponding author at: UNC School of Medicine, University of North Carolina, 4008 Burnett Womack Building, CB 7228, United States. Fax: +1 919 9660369. anthchar@med.unc.edu (A. Charles).

Conflict of interest

The authors declare that they have no conflict of interest.
} 


\section{Introduction}

Cardiovascular disease (CVDs) is the leading cause of death and major cause of disability worldwide [1,2]. An estimated 17.5 million people died from CVDs in 2012, representing $31 \%$ of all global deaths. Of these deaths, an estimated 7.4 million were due to coronary heart disease and 6.7 million were due to stroke. CVDs are a group of disorders of the heart and blood vessels and include coronary heart disease with or without heart failure, dysrhythmias, cardiomyopathy; cerebrovascular disease including aneurysmal diseases; peripheral arterial disease; rheumatic heart disease- damage to the heart muscle and heart valves; congenital heart disease- malformations of heart structure existing at birth and deep vein thrombosis and pulmonary embolism $[3,4]$. With the increase in longevity of the US population, there is a concomitant rise in the number of people with cardiovascular comorbidities that may also have surgical needs.

The association between burn and cardiovascular dysfunction has long been established $[5,6]$ with patients presenting with "burn Shock". This is a complex combination of hypovolemic and cardiogenic shock with the later onset of a septic component following burn. It leads to a profound, persistent and unique derangement of cardiovascular function [7]. Derangements in cardiac function can last for two years in pediatric patients with burns encompassing over thirty percent of their total body surface area [8]. Factors that influence outcomes following burn are well defined and they include, percent total body surface area burn, presence of inhalational injury, the extremes of age and burn wound related sepsis. However, the effect of the resultant physiologic changes on burn patients with pre-existing cardiovascular diseases is as yet undefined.

There is anecdotal evidence to suggest that cardiovascular comorbidities may adversely alter outcomes and indirect evidence suggests that malperfusion contributes immensely to increased mortality in patients with cardiac comorbidities following traumatic injury but specific evidence in the burn injured population is limited. In this study, we sought to characterize the relationship between pre-existing cardiovascular comorbidities and burn outcomes. We hypothesize that patients with preexisting cardiovascular disease will have an increased morbidity and mortality following burn.

\section{Methods}

This is a retrospective study of all burn patients admitted to the North Carolina Jaycee Burn Center from 2002 to 2012. The North Carolina Jaycee Burn Center at University of North Carolina is an American Burn Association verified pediatric and adult center. Established in 1981 and the center averages more than 1200 acute admissions per year. The burn center is a 36-bed single unit facility.

The medical records of adult subjects (>18years) identified by the UNC Burn database query were reviewed to verify baseline demographic data, injury characteristics, and provide detailed information on medical comorbidities and some complications (i.e. cardiac). Injury characteristics of interest included burn etiology, \%TBSA burn, presence of inhalation injury, and ventilation status on admission to the burn center. Inhalation injury diagnosis was 
based on history, physical examination, and/or bronchoscopic examination. Cardiac complications include arrhythmias, myocardial infarction, congestive heart failure, or cardiac arrest that occurred after admission. Sepsis is recoded in the burn registry based on ICD-9 codes. The cause of death for patients in the cohort is not recorded in the burn registry.

To examine the effect of baseline medical comorbidities on outcome, a Charlson Comorbidity Index (CCI) score was calculated for each patient. The standardized Charlson Index has been reported to accurately predict the probability of mortality within 1year for a number of medical conditions $[9,10]$. The score is the weighted sum of comorbid conditions. There are 17 comorbid conditions included in the score and each is assigned a weight from 1 to 6 points. The weighted sum of all comorbid conditions is the patient's Charlson score. (Table 1) The CCI was also modified for this study to exclude cardiovascular disease in order to elicit the independent effect of cardiovascular disease on burn outcome. Pre-existing comorbidities were identified utilizing the National Trauma Data Bank (NTDB) from the American College of Surgeons Committee on Trauma. It includes 27 different preinjury comorbidities.

The primary outcomes of interest in this study were mechanical ventilation and in-hospital mortality. Baseline patient and injury characteristics were compared between groups (CVD/no CVD) using Analysis of Variance for continuous variables and chi-squared for discrete variables. We employed both univariate and multivariate models to determine the relative influence of preexisting CVDs after controlling for covariates. To estimate the incidence rate ratios for mechanical ventilation and mortality, we used a multivariate Poisson regression model controlling for pertinent confounders (age, TBSA, mechanism of injury, presence of inhalation injury, comorbidities using CCI, and sepsis). Stata/MP (Version 12) (Stata Corp, College Station, TX) was used for all data management and statistical analysis. The University of North Carolina Institutional Review Board approved this study.

\section{Results}

A total of 5332 adult patients admitted during the study period and included in our analysis. The median age was $41.8(\mathrm{IQR}=29.6-54.2)$ years with a male predominance at $3859(72 \%)$. Caucasians made up 2995 (56\%) of the population. The most common burn mechanism was flame $(n=2936,55 \%)$ followed by scald injury $(n=2006,38 \%)$. The overall median \%TBSA for this population was 5 (IQR=2-11) \%. (Table 2) The mean hospital, ICU length ofstay, and days of mechanical ventilation was $15.0 \pm 27$.3days, $17.4 \pm 35$.8days, and $26.1 \pm 38$.4days respectively. Burn patients with at least one comorbidity represented $55 \%$ of the population $(n=2915)$. A total of $428(8 \%)$ patients had pre-existing cardiovascular disease prior to burn. For this subset of patients, cardiovascular diseases present were coronary artery disease $(\mathrm{n}=272,63 \%)$, cardiac arrhythmias $(\mathrm{n}=145,33 \%)$, myocardial infarction $(\mathrm{n}=121,28 \%)$, congestive heart failure $(n=127,30 \%)$, stroke $(n=83,19 \%)$, peripheral vascular disease $(\mathrm{n}=63,15 \%)$, and heart valve abnormality $(\mathrm{n}=70,16 \%)$. In the entire cohort, known risk factors for cardiovascular disease included concurrent smokers $(\mathrm{n}=1382,26 \%)$, hypertension $(n=1037,19 \%)$, diabetes $(n=603,11 \%)$, and obesity $(n=139,3 \%)$. A total of $181(3 \%)$ patients experienced in-hopsital cardiac complications. 


\subsection{Cardiovascular disease}

A bivariate analysis was performed to compare patients with preexisting cardiovascular disease (PCVD) and without pre-existing cardiovascular disease (NCVD). The median age for PCVD vs NCVD was 63.4 (IQR=54.5-73.5)years and 40.0 (IQR=28.7-51.7)years, respectively $(\mathrm{p}<0.001)$. (Table 3$)$ There was a no significant difference between the two groups based on \%TBSA, race or gender. PCVD patients had a higher modified CCI score $(1.65 \pm 1.61)$ compared to NCVD patients $(0.35 \pm 0.92, \mathrm{p}<0.001)$. For risk factors, preexisting cardiovascular disease patients have significantly higher rates of smoking (33\% vs $25 \%$, $\mathrm{p}<0.001$ ), hypertension ( $53 \%$ vs. $17 \%, \mathrm{p}<0.001$ ), diabetes ( $42 \%$ vs $9 \%, \mathrm{p}<0.001)$, and obesity ( $7 \%$ vs $2 \%, \mathrm{p}<0.001$ ) compared to NCVD patients. Approximately, $15 \%$ (vs. 2\%) patients with PVD had cardiac complications and 9\% (vs. 3\%) developed sepsis ( $\mathrm{p}<0.001)$. Inhalation injury was higher in PCVD patients compared to NCVD patients (14\% vs. 9\%, $\mathrm{p}<0.001)$. The crude mortality rate was higher for patients with PCVD (15\% vs. $4 \%$, $\mathrm{p}<0.001)$. Patients with PCVD had statistically significant increase in hospital-LOS (25.0 \pm 36.9 days vs. $14.1 \pm 26.1$ days, $\mathrm{p}<0.001)$, ICU-LOS stay (13.6 \pm 32.1 days vs. $5.9 \pm 22.4$ days, $\mathrm{p}<0.001)$, and ventilator days (8.8days vs. 3.8days, $\mathrm{p}<0.001)$ compared to NCVD patients.

A multivariate Poisson regression model was utilized to determine the incidence risk of intensive care unit admission and burn mortality in the PCVD vs. CVD subgroups after adjusting for significant covariates. Patients with PCVD werein the intensive care unit 30\% more (IRR=1.30 (NCVD reference group), 95\% CI=1.19-1.43). (Table 4) The cumulative risk for patients with preexisting cardiovascular disease for ICU admission was higher than NRD patients over the first 100days of hospital stay. (Fig. 1) The unadjusted incidence of mortality for patients with PCVD is 3.41 times that of NCVD patients (IRR=3.41 (NCVD reference group), 95\% $\mathrm{CI}=2.63-4.42$ ). However, the risk ratio for mortality is $46 \%$ higher (IRR=1.46 (NCVD reference group), 95\% confidence interval: 1.13-1.90) after adjustment of patient demographics, injury characteristics, and in-hospital complications (Table 5, Fig. 2). When patients are group based on presence of cardiovascular disease, patients with preexisting cardiovascular disease were at higher cumulative risk of mortality than noncardiovascular disease patients over the length of hospitalization (Fig. 2).

\section{Discussion}

In this study, we have shown that burn patient with preexisting cardiovascular comorbidities have an increased intensive care unit admission and significantly higher risk of mortality compared to burn patients without pre-existing cardiovascular diseases.

Studies in the general trauma population reveal similar findings to ours. Ferraris et al. studied the relationship between preexisting cardiac disease and mortality in 5971 trauma patients over a 5-year period. The study revealed that the most significant cardiovascular risk of trauma death were preinjury use of Coumadin, a surrogate for dysrhythmias or deep vein thrombosis/pulmonary embolism ( $\mathrm{OR}=2.309)$, and congestive heart failure $(\mathrm{OR}=2.060)$. The mortality rate was highest in patients with a combination of these cardiovascular factors, especially if the patient was on Coumadin prior to admission [11]. In a study examining burn outcomes associated with comorbidities over a shorter time period, Akhtar et al. also found 
that cardiac disease had the highest mortality rate $(10.5 \%)$ compared to other chronic diseases but a similar length of hospital stay (5weeks) [12].

The only prior study that specifically examined mortality following burn in patients with and without prior CVD reviewed 2477 consecutive burn admissions and observed the incidence of cardiac complications in patients with burns. Forty-six percent of patients with CVD prior to burn experienced at least one cardiac event during the hospitalization. Cardiac complications include arrhythmias, hypotension, congestive heart failure, myocardial infarction, and cardiac arrest. For patients with history valvular disease, the average burn size (\%TBSA) ranged from 16 to $29 \%$ that had cardiac dysfunction. The mean \% TBSA for those who suffered a myocardial infarction was $30.3 \%$. The mortality rate for those patients with cardiac history was $30 \%$. In comparison to patients without a cardiac history, the inhospital mortality rate following myocardial infarction was 3.5-4 times higher for those with a cardiac history [13].

The specific drivers and pathophysiologic mechanisms that predispose burn patients with PCVD to increased mortality are not well delineated as we cannot directly attribute the cause of death to cardiovascular disease without an autopsy. However, it is clearly established that severe burn is complicated by a surge of plasma catecholamines that mediate the profound hypermetabolic response. The elevated levels of plasma catecholamines instigate cardiac stress after burn. [14] The effects of a sustained release of large amounts of circulating catecholamines are detrimental to the myocardium. The resultant increased sympathetic drive results in anticipated increased myocardial oxygen delivery and myocardial oxygen consumption. In the presence of preexisting cardiovascular disease, such as coronary arterial disease or cardiac arrhythmias, the resultant cardiac response may be inadequate to meet needs leading to local myocardial hypoxia, focal degeneration of the myocardium, cardiac instability and cardiac death, and physiologic exhaustion [15-17].

In this study, we controlled for inhalation injury, we recognize that the presence of identifiable (carbon monoxide) and other non-identifiable toxins creates a hypoxic intracellular environment that results in myocardial damage, which can lead to dysrhythmias, new onset or worsening congestive heart failure, hypotension, or acute myocardial infarction [18]. In addition, the massive fluid shifts associated with burn resuscitations may result in additional life-threatening complications as pulmonary edema and abdominal compartment syndrome that may predispose burn patients with cardiovascular disease to increased risk of death [19-21].

The pathophysiology of cardiac dysfunction in burns is increasingly recognized, however, evidence guiding the treatment remains elusive. Strategies to mitigate the poor outcomes in the subset of burn patients with preexisting cardiovascular disease should include early recognition of the type of preexisting CVD and the institution of critical care. The prevention of burn shock should be an early goal. Balanced fluid resuscitation with hemodynamic end points to avoid over-resuscitation and perhaps the use of goal directed resuscitation using invasive or non-invasive adjuncts such as the pulmonary artery or other thermodilution cardiac monitor catheters or cardiac ultrasound, respectively. 
Following the resuscitation period, the attenuation of the catecholamine response should be attempted. During this phase, complications such as cardiac arrhythmias, myocardial infarctions, and cardiac instability may manifest and the use of B-blockers may be considered. Previous studies in burn patients have shown significant promise with the use of B-Blockers to attenuate the hypermetabolic response and reverse muscle protein catabolism [22]. The use of propranolol also improved cardiac function and lowered the heart rate while maintaining adequate cardiac output [23]. Studies on use of B-blockers in adults have shown improved burn outcomes such as decreased mortality rate and improved wound healing [24]. In terms of burn mortality, patients who were able to achieve greater cardiac output and oxygen delivery had better survival outcomes [25].

The limitations of this study are those inherent to any retrospective study. As a large burn center, presentation bias may have affected our patient mix as more complicated burn were sent to our center. Though this is a single center study, our sample size was robust enough not to affect the generalizability of our findings. The pre-existing comorbidities were abstracted from documented diagnosed based on ICD-9 codes and hence subject to interpretation. All cardiovascular diseases were groups were categorized as one entity and the individual contribution of each individual cardiovascular disease and its weight on burn mortality was not analyzed. The contribution of frailty and its effect on to outcome was not evaluated. An alternative would be the use of the APACHE score on admission which gives a better indication of health status on admission but the APACHE score has not been validated in a large cohort of burn patients. Furthermore, the Charlson comorbidity index was originally based on the predictive power to estimate mortality in medicine patients and was never intended to generalizations to surgical or burn cohort.

\section{Conclusion}

Preexisting cardiovascular disease significantly increases the risk of intensive care unit admission and burn mortality. Our findings re-emphasizes the need for the inclusion of comorbidities in any burn mortality prediction models so as to better inform burn mortality prognostication. Understanding of the pathophysiology of cardiovascular dysfunction and how the additional stress of burn can affect the underlying cardiovascular disease process is critical to the successful patient management in this burn population to reduce mortality.

\section{References}

1. Lopez, AD., Mathers, CD., Ezzati, M., Jamison, DT., Murray, CJL. Measuring the global burden of disease and risk factors, 1990-2001. In: Lopez, AD., et al., editors. Global burden of disease and risk factors. New York: Oxford University Press; 2006.

2. Murray, CJL., Lopez, AD. The global burden of disease: a comparative assessment of mortality and disability from diseases, injuries, and risk factorsin 1990 and projected to 2020. Vol. 1. Cambridge, Mass: Harvard University Press; 1996.

3. Mendis, S., Puska, P., Norrving, B. World Health Organization. World Health Organization in collaboration with the World Heart Federation and the World Stroke Organization. 2011. Global atlas on cardiovascular disease prevention and control; p. 3-18.

4. World Health Organization-Media Centre. [Accessed 27 June 2016] Cardiovascular disease Fact sheet. Reviewed June 2016. http://www.who.int/mediacentre/factsheets/fs317/en/ 
5. Horton JW. Left ventricular contractile dysfunction as a complication of thermal injury. Shock. 2004; 22:495-507. [PubMed: 15545819]

6. Baxter CR, Cook WA, Shires ST. Serum myocardial depressant factor of burn shock. Surg Forum. 1966; 17:1-2. [PubMed: 5920908]

7. Kramer, GC., Lund, K., Beckum, OK. Pathophysiology of burn shock and burn edema. In: Herndon, DN., editor. Total burn care. 3. Philadephia, PA: Elsevier Health Sciences; 2007. p. 93-106.

8. Williams FN, Herndon DN, Suman OE, Lee JO, Norbury WB, Branski LK, et al. Changes in cardiac physiology after sever burn injury. J Burn Care Res. 2011; 32(2):269-74. doi:http://dx.doi.org/ 10.1097/BCR.0b013e31820aafcf. [PubMed: 21228708]

9. Charlson ME, Pompei P, Ales KL, MacKenzie CR. A new method of classifying prognostic comorbidity in longitudinal studies: development and validation. J Chronic Dis. 1987; 40:373-83. [PubMed: 3558716]

10. Needham DM, Scales DC, Laupacis A, Pronovost PJ. A systematic review of the Charlson comorbidity index using Canadian administrative databases: a perspective on risk adjustment in critical care research. J Crit Care. 2005; 20:12-9. [PubMed: 16015512]

11. Ferraris VV, Ferraris SP, Saha SP. The relationship between mortality and preexisting cardiac disease in 5,971 trauma patients. J Trauma. 2010 Sep; 69(3):645-52. doi:http://dx.doi.org/ 10.1097/TA.0b013e3181d8941d. [PubMed: 20526211]

12. Akhtar MS, Ahmad I, Khan AH, Khurram FM, Haq A, Basari R. Burn injury associated with comorbidities: impact on the outcome. Indian J Burns. 2014; 22:51-5.

13. Goff DR, Purdue GF, Hunt JL, Cochran RP. Cardiac disease and the patient with burns. J Burn Care Rehabil. 1990; 11:305-7. [PubMed: 2401685]

14. Pereira CT, Herndon DN. The pharmacologic modulation of the hypermetabolic response to burns. Adv Surg. 2005; 39:245-61. [PubMed: 16250555]

15. Cuthbertson DP, Angeles Valero Zanuy MA, Leon Sanz ML. Post-shock metabolic response 1942. Nutr Hosp. 2001; 16:176-82. [PubMed: 11708288]

16. Schultz AM, Werba A, Wolrab C. Early cardiorespiratory patterns in severely burned patients with concomitant inhalationinjury. Burns. 1997; 23:421-5. [PubMed: 9426912]

17. Coumel P. Autonomic influences in atrial tachycardias. J Cardiovasc Electrophysiol. 1996; 7:999_ 1007. [PubMed: 8894942]

18. Williams A, Lewis RW [121_TD\$DIFF]2nd, Kealey GP. Carbon monoxide poisoning and myocardial ischaemia in patients with burns. J Burn Care Rehabil. 1992; 13:210-3. [PubMed: 1587919]

19. Deitch EA. The management of burns. N Engl J Med. 1990; 323:1249-53. [PubMed: 2120587]

20. Hughes KR, Armstrong RF, Brough MD, Parkhouse N. Fluid requirements of patients with burns and inhalation injuries in an intensive care unit. Intensive Care Med. 1989; 15:464-6. [PubMed: 2600291]

21. Abu-Sittah GS, Sarhane KA, Dibo SA, Ibrahim A. Cardiovascular dysfunction in burns: review of the literature. Ann Burns Fire Disasters. 2012; 25(1):26-37. [PubMed: 23012613]

22. Herndon DN, Hart DW, Wolf SE, Chinkes DL, Wolfe RR. Reversal of catabolism by beta blockade after severe burns. N Engl J Med. 2001; 345:1223-9. [PubMed: 11680441]

23. Herndon DN, Barrow RE, Rutan TC, Minifee O, Jahoor F, Wolfe RR. Effect of propranolol administration on haemodynamics and metabolic responses of burned paediatric patients. Ann Surg. 1988; 208:484-92. [PubMed: 3052328]

24. Arbabi S, Ahrns KS, Wahl WL, Hemmila MR, Wang SC, Brandt MM, et al. Beta-blocker use is associated with improved outcomes in adult burn patients. J Trauma. 2004; 56:265-71. [PubMed: 14960966]

25. Holm C, Melcer H, Horbrand F, Worl HH, von Donnersmarck GH, Muhlbauer W. Haemodynamic and oxygen transport responses in survivors and non-survivors and non-survivors following thermal injury. Burns. 2000; 26:25-33. [PubMed: 10630316] 


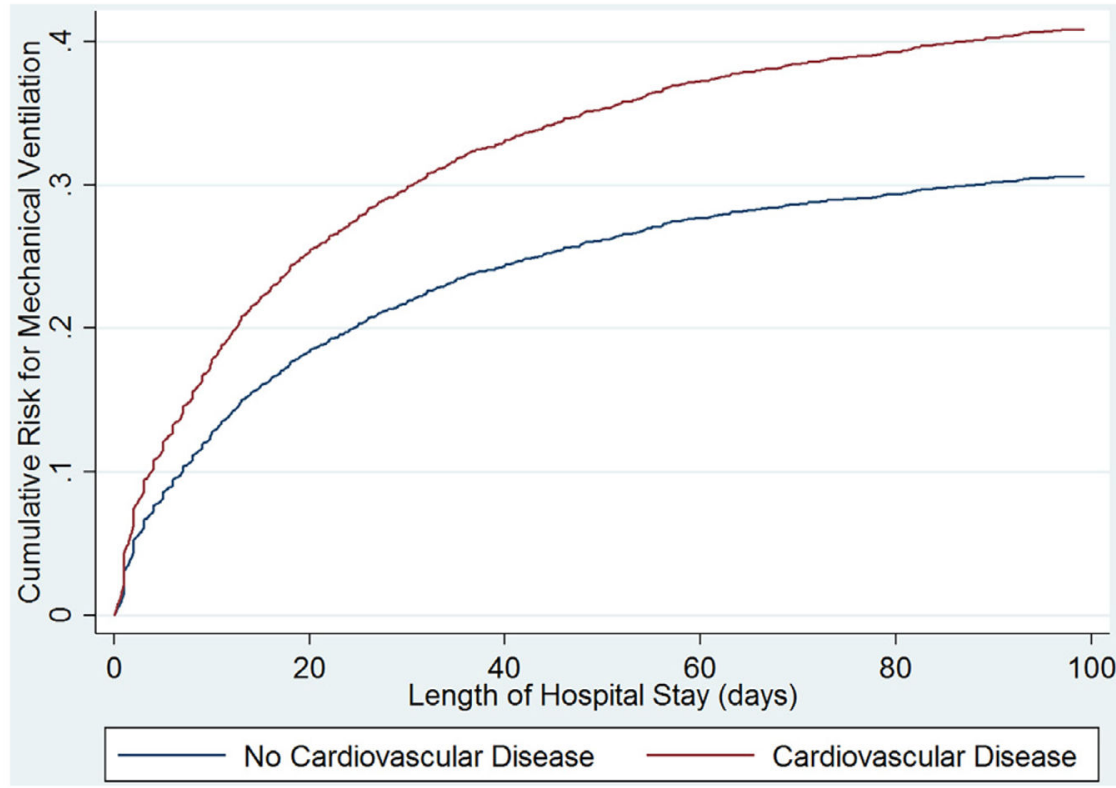

Fig. 1.

Cumulative risk for intensive care unit admission in patients with and without pre-existing cardiovascular disease. 


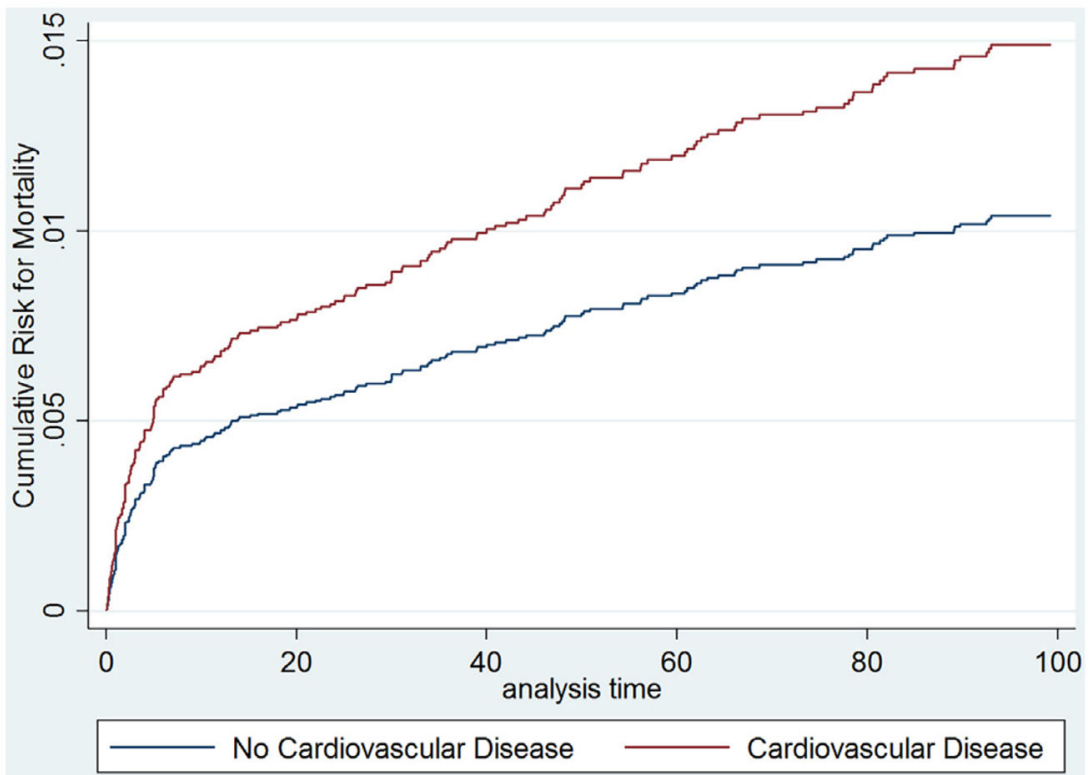

Fig. 2.

Cumulative risk for mortality in patients with and without pre-existing cardiovascular disease. 


\section{Table 1}

Charlson comorbidity index score system.

\begin{tabular}{|c|c|}
\hline Comorbidity & Score \\
\hline Myocardial infarction & 1 \\
\hline Congestive heart failure & 1 \\
\hline Peripheral vascular disease & 1 \\
\hline Cerebrovascular disease & 1 \\
\hline Dementia & 1 \\
\hline Chronic pulmonary disease & 1 \\
\hline Rheumatologic disease & 1 \\
\hline Peptic ulcer disease & 1 \\
\hline Mild liver disease & 1 \\
\hline Diabetes without chronic complications & 1 \\
\hline Diabetes with chronic complications & 2 \\
\hline Hemiplegia or paraplegia & 2 \\
\hline Renal disease & 2 \\
\hline Any malignancy, including leukemia and lymphoma & 2 \\
\hline Moderate or severe liver disease & 3 \\
\hline AIDS/HIV & 6 \\
\hline Metastatic solid tumor & 6 \\
\hline Maximum comorbidity score & 33 \\
\hline
\end{tabular}

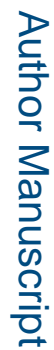

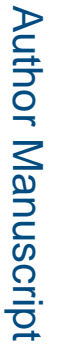

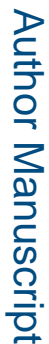




\section{Table 2}

Patient characteristics.

\begin{tabular}{|lll|}
\hline Patient characteristics $(\mathbf{n}=\mathbf{7 6 4 0})$ & $\boldsymbol{n}$ & Mean $( \pm$ SD $)$ or $\%$ \\
\hline Gender & & \\
$\quad$ Male & 5244 & $69 \%$ \\
$\quad$ Female & 2396 & $31 \%$ \\
Age & & \\
$\quad$ Overall & $32.0( \pm 22.4)$ \\
Race & & \\
$\quad$ White & 3896 & $51 \%$ \\
$\quad$ Other & 3742 & $49 \%$ \\
\end{tabular}

Type of burn

Other

$480 \quad 6 \%$

Inhalation

Inhalation injury

$562 \quad 7 \%$

No inhalation injury

$7078 \quad 93 \%$

Charlson comorbidity index (CCI)

Overall mean

$7640 \quad 0.906864( \pm 1.4)$

$40 \%$

Cardiovascular disease

Pre-existing

$432 \quad 6 \%$

None

$7209 \quad 94 \%$

TBSA

Overall mean

$7640 \quad 8.6( \pm 12.0)$

By gender

Male

$5244 \quad 9.0( \pm 12.6)$

Female

$2396 \quad 7.7( \pm 10.7)$

Survival

Overall

$7274 \quad 96 \%$

By gender

Male

Female

$5017 \quad 96 \%$

$2257 \quad 94 \%$

By Race

White $\quad 3699 \quad 96 \%$

Other

ICU stay

Overall mean

$2512 \quad 5.1( \pm 20.2)$

Hospital stay

Overall mean

$12.7( \pm 24.7)$

Mechanical ventilation 


\begin{tabular}{|cll|}
\hline Patient characteristics $(\mathbf{n}=\mathbf{7 6 4 0})$ & $\boldsymbol{n}$ & Mean $( \pm$ SD) or $\%$ \\
\hline Overall mean & 956 & $3.2( \pm 15.8)$ \\
\hline
\end{tabular}

TBSA=total burn surface area 


\section{Table 3}

Bivariate analysis of burn patients (with and without preexisting cardiovascular disease).

\begin{tabular}{|c|c|c|c|}
\hline & Cardiovascular disease $(n=428)$ & No cardiovascular disease $(n=4903)$ & p-Value \\
\hline Age & $63.4(\mathrm{IQR}=54.5-73.5)$ & $40.0(\mathrm{IQR}=28.7-51.7)$ & $<0.001$ \\
\hline TBSA & $10.2(\mathrm{IQR}=2-13)$ & $9.3(\mathrm{IQR}=2-10)$ & 0.115 \\
\hline $\mathrm{mCCI}$ & $1.65( \pm 1.61)$ & $0.35( \pm 0.92)$ & $<0.001$ \\
\hline Race & & & 0.060 \\
\hline White & $259(61 \%)$ & $2735(56 \%)$ & \\
\hline Other & $169(39 \%)$ & $2165(44 \%)$ & \\
\hline Gender & & & 0.418 \\
\hline Male & $317(74 \%)$ & $3542(72 \%)$ & \\
\hline Female & $111(26 \%)$ & $1361(28 \%)$ & \\
\hline Mechanism & & & 0.014 \\
\hline Flame & $264(62 \%)$ & $2671(55 \%)$ & \\
\hline Scald & $136(32 \%)$ & $1870(38 \%)$ & \\
\hline Other & $24(6 \%)$ & $325(7 \%)$ & \\
\hline Smoker & $142(33 \%)$ & $1240(25 \%)$ & $<0.001$ \\
\hline HTN & $228(53 \%)$ & $810(17 \%)$ & $<0.001$ \\
\hline Obesity & $31(7 \%)$ & $118(2 \%)$ & $<0.001$ \\
\hline Diabetes & $180(42 \%)$ & $422(9 \%)$ & $<0.001$ \\
\hline Cardiac complications & $64(15 \%)$ & $117(2 \%)$ & $<0.001$ \\
\hline Sepsis & $37(9 \%)$ & $128(3 \%)$ & $<0.001$ \\
\hline Inhalation injury & $62(14 \%)$ & $461(9 \%)$ & 0.001 \\
\hline Crude mortality rate & $65(15 \%)$ & $216(4 \%)$ & $<0.001$ \\
\hline ICU LOS & $13.6( \pm 32.1)$ & $5.9( \pm 22.4)$ & $<0.001$ \\
\hline Length of hospital stay & $25.0( \pm 36.9)$ & $14.1( \pm 26.1)$ & $<0.001$ \\
\hline Mechanical ventilation & $8.8( \pm 29.1)$ & $3.8( \pm 16.9)$ & $<0.001$ \\
\hline
\end{tabular}

TBSA=total body surface area.

$\mathrm{mCCI}=$ Charlson comorbidity index excluding preexisting cardiovascular diseases.

HTN=Hypertension.

ICU LOS=intensive care unit length of stay. 
Table 4

Multivariate Poisson regression for being in ICU admission in burn cohort.

\begin{tabular}{|llr|}
\hline Variable & Adjusted incidence rate ratio, 95\% confidence interval (CI) & p-Value \\
\hline Age & $1.01(1.00-1.01)$ & $<0.001$ \\
TBSA & $1.01(1.01-1.02)$ & $<0.001$ \\
Mechanism & & \\
Flame (reference group) & & \\
Scald & $0.61(0.55-0.67)$ & $<0.001$ \\
Other & $1.54(1.39-1.71)$ & $<0.001$ \\
mCCI & $1.05(1.02-1.08)$ & $<0.001$ \\
Inhalation injury & $1.91(1.80-2.03)$ & $<0.001$ \\
Sepsis & $1.17(1.08-1.28)$ & $<0.001$ \\
Cardiac Complications & $1.29(1.17-1.42)$ & $<0.001$ \\
Cardiovascular Disease & $1.30(1.18-1.43)$ & $<0.001$ \\
\hline
\end{tabular}

TBSA=total body surface area.

mCCI=Charlson comorbidity index excluding cardiovascular diseases. 
Table 5

Multivariate Poisson regression for in-hospital mortality in burn cohort.

\begin{tabular}{|llr|}
\hline Variable & Adjusted incidence rate ratio, 95\% confidence interval (CI) & p-Value \\
\hline Age & $1.04(1.03-1.05)$ & $<0.001$ \\
TBSA & $1.04(1.04-1.05)$ & $<0.001$ \\
Mechanism & & \\
$\quad$ Flame (reference group) & & \\
$\quad$ Scald & $0.61(0.43-0.86)$ & \\
Other & $1.44(0.79-2.60)$ & 0.005 \\
LOS & $0.98(0.97-0.99)$ & 0.230 \\
Mechanical ventilation & $1.02(1.00-1.04)$ & 0.011 \\
mCCI & $1.11(1.03-1.20)$ & 0.013 \\
Inhalation injury & $1.81(1.46-2.24)$ & 0.008 \\
Sepsis & $3.06(2.18-4.29)$ & $<0.001$ \\
Cardiac complications & $1.60(1.16-2.22)$ & $<0.001$ \\
Cardiovascular disease & $1.46(1.13-1.90)$ & 0.004 \\
\hline
\end{tabular}

TBSA=total body surface area.

LOS=length of hospital stay.

$\mathrm{mCCI}=$ Charlson comorbidity index excluding cardiovascular diseases. 